\title{
Remarks on Branching-Extinction Evolutionary Cycles
}

Fabio Dercole (fabio.dercole@polimi.it)

\section{Approved by \\ Ulf Dieckmann (dieckmann@iiasa.ac.at) \\ Project Leader, Adaptive Dynamics Network \\ December 2003}




\section{IIASA STUDIES IN ADAPTIVE DYNAMICS No. 75}

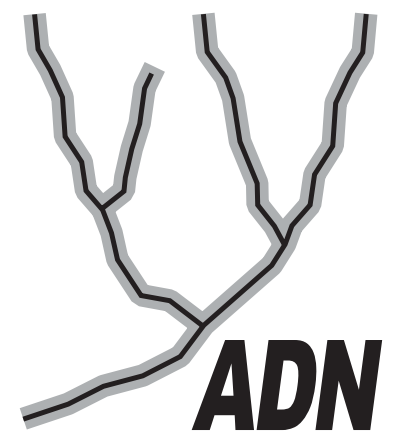

The Adaptive Dynamics Network at IIASA fosters the development of new mathematical and conceptual techniques for understanding the evolution of complex adaptive systems.

Focusing on these long-term implications of adaptive processes in systems of limited growth, the Adaptive Dynamics Network brings together scientists and institutions from around the world with IIASA acting as the central node.

Scientific progress within the network is collected in the IIASA Studies in Adaptive Dynamics series.
No. 1 Metz JAJ, Geritz SAH, Meszéna G, Jacobs FJA, van Heerwaarden JS: Adaptive Dynamics: A Geometrical Study of the Consequences of Nearly Faithful Reproduction. IIASA Working Paper WP-95-099 (1995). van Strien SJ, Verduyn Lunel SM (eds): Stochastic and Spatial Structures of Dynamical Systems, Proceedings of the Royal Dutch Academy of Science (KNAW Verhandelingen), North Holland, Amsterdam, pp. 183-231 (1996).

No. 2 Dieckmann U, Law R: The Dynamical Theory of Coevolution: A Derivation from Stochastic Ecological Processes. IIASA Working Paper WP-96-001 (1996). Journal of Mathematical Biology 34:579-612 (1996).

No. 3 Dieckmann U, Marrow P, Law R: Evolutionary Cycling of Predator-PreyInteractions: Population Dynamics and the Red Queen. IIASA Preprint (1995). Journal of Theoretical Biology 176:91-102 (1995).

No. 4 Marrow P, Dieckmann U, Law R: Evolutionary Dynamics of Predator-Prey Systems: An Ecological Perspective. IIASA Working Paper WP-96-002 (1996). Journal of Mathematical Biology 34:556-578 (1996).

No. 5 Law R, Marrow P, Dieckmann U: On Evolution under Asymmetric Competition. IIASA Working Paper WP-96-003 (1996). Evolutionary Ecology 11:485-501 (1997).

No. 6 Metz JAJ, Mylius SD, Diekmann O: When Does Evolution Optimize? On the Relation Between Types of Density Dependence and Evolutionarily Stable Life History Parameters. IIASA Working Paper WP-96-004 (1996).

No. 7 Ferrière R, Gatto M: Lyapunov Exponents and the Mathematics of Invasion in Oscillatory or Chaotic Populations. Theoretical Population Biology 48:126-171 (1995).

No. 8 Ferrière R, Fox GA: Chaos and Evolution. IIASA Preprint (1996). Trends in Ecology and Evolution 10:480485 (1995).

No. 9 Ferrière R, Michod RE: The Evolution of Cooperation in Spatially Heterogeneous Populations. IIASA Working Paper WP-96-029 (1996). The American Naturalist 147:692717 (1996).

No. 10 van Dooren TJM, Metz JAJ: Delayed Maturation in Temporally Structured Populations with Non-Equilibrium Dynamics. IIASA Working Paper WP-96-070 (1996). Journal of Evolutionary Biology 11:41-62 (1998).
No. 11 Geritz SAH, Metz JAJ, Kisdi É, Meszéna G: The Dynamics of Adaptation and Evolutionary Branching. IIASA Working Paper WP-96-077 (1996). Physical Review Letters 78:2024-2027 (1997).

No. 12 Geritz SAH, Kisdi É, Meszéna G, Metz JAJ: Evolutionary Singular Strategies and the Adaptive Growth and Branching of the Evolutionary Tree. IIASA Working Paper WP-96-114 (1996). Evolutionary Ecology 12:35-57 (1998).

No. 13 Heino M, Metz JAJ, Kaitala V: Evolution of Mixed Maturation Strategies in Semelparous Life-Histories: The Crucial Role of Dimensionality of Feedback Environment. IIASA Working Paper WP-96-126 (1996). Philosophical Transactions of the Royal Society of London Series B 352:1647-1655 (1997).

No. 14 Dieckmann U: Can Adaptive Dynamics Invade? IIASA Working Paper WP-96-152 (1996). Trends in Ecology and Evolution 12:128-131 (1997).

No. 15 Meszéna G, Czibula I, Geritz SAH: Adaptive Dynamics in a 2-Patch Environment: A Simple Model for Allopatric and Parapatric Speciation. IIASA Interim Report IR-97-001 (1997). Journal of Biological Systems 5:265-284 (1997).

No. 16 Heino M, Metz JAJ, Kaitala V: The Enigma of Frequency-Dependent Selection. IIASA Interim Report IR97-061 (1997). Trends in Ecology and Evolution 13:367-370 (1998).

No. 17 Heino M: Management of Evolving Fish Stocks. IIASA Interim Report IR-97-062 (1997). Canadian Journal of Fisheries and Aquatic Sciences 55:1971-1982 (1998).

No. 18 Heino M: Evolution of Mixed Reproductive Strategies in Simple Life-History Models. IIASA Interim Report IR-97063 (1997).

No. 19 Geritz SAH, van der Meijden E, Metz JAJ: Evolutionary Dynamics of Seed Size and Seedling Competitive Ability. IIASA Interim Report IR-97-071 (1997). Theoretical Population Biology 55:324-343 (1999).

No. 20 Galis F, Metz JAJ: Why Are There So Many Cichlid Species? On the Interplay of Speciation and Adaptive Radiation. IIASA Interim Report IR-97-072 (1997). Trends in Ecology and Evolution 13:1-2 (1998). 
No. 21 Boerlijst MC, Nowak MA, Sigmund K: Equal Pay for all Prisoners/ The Logic of Contrition. IIASA Interim Report IR-97-073 (1997). American Mathematical Society Monthly 104:303-307 (1997). Journal of Theoretical Biology 185:281-293 (1997).

No. 22 Law R, Dieckmann U: Symbiosis Without Mutualism and the Merger of Lineages in Evolution. IIASA Interim Report IR-97-074 (1997). Proceedings of the Royal Society of London Series B 265:1245-1253 (1998).

No. 23 Klinkhamer PGL, de Jong TJ, Metz JAJ: Sex and Size in Cosexual Plants. IIASA Interim Report IR-97-078 (1997). Trends in Ecology and Evolution 12:260-265 (1997).

No. 24 Fontana W, Schuster P: Shaping Space: The Possible and the Attainable in RNA Genotype-Phenotype Mapping. IIASA Interim Report IR-98-004 (1998). Journal of Theoretical Biology 194:491-515 (1998).

No. 25 Kisdi É, Geritz SAH: Adaptive Dynamics in Allele Space: Evolution of Genetic Polymorphism by Small Mutations in a Heterogeneous Environment. IIASA Interim Report IR-98-038 (1998). Evolution 53:993-1008 (1999).

No. 26 Fontana W, Schuster P: Continuity in Evolution: On the Nature of Transitions. IIASA Interim Report IR-98-039 (1998). Science 280:1451-1455 (1998).

No. 27 Nowak MA, Sigmund K: Evolution of Indirect Reciprocity by Image Scoring/ The Dynamics of Indirect Reciprocity. IIASA Interim Report IR-98-040 (1998). Nature 393:573-577 (1998). Journal of Theoretical Biology 194:561574 (1998).

No. 28 Kisdi É: Evolutionary Branching Under Asymmetric Competition. IIASA Interim Report IR-98-045 (1998). Journal of Theoretical Biology 197:149-162 (1999).

No. 29 Berger U: Best Response Adaptation for Role Games. IIASA Interim Report IR-98-086 (1998).

No. 30 van Dooren TJM: The Evolutionary Ecology of Dominance-Recessivity. IIASA Interim Report IR-98-096 (1998). Journal of Theoretical Biology 198:519-532 (1999).

No. 31 Dieckmann U, O'Hara B, Weisser W: The Evolutionary Ecology of Dispersal. IIASA Interim Report IR-98-108 (1998). Trends in Ecology and Evolution 14:88-90 (1999).

No. 32 Sigmund K: Complex Adaptive Systems and the Evolution of Reciprocation. IIASA Interim Report IR-98-100 (1998). Ecosystems 1:444-448 (1998).

No. 33 Posch M, Pichler A, Sigmund K: The Efficiency of Adapting Aspiration Levels. IIASA Interim Report IR-98103 (1998). Proceedings of the Royal Society London Series B 266:1427-1435 (1999).

No. 34 Mathias A, Kisdi É: Evolutionary Branching and Coexistence of Germination Strategies. IIASA Interim Report IR-99-014 (1999).

No. 35 Dieckmann U, Doebeli M: On the Origin of Species by Sympatric Speciation. IIASA Interim Report IR-99-013 (1999). Nature 400:354-357 (1999).

No. 36 Metz JAJ, Gyllenberg M: How Should We Define Fitness in Structured Metapopulation Models? Including an Application to the Calculation of Evolutionarily Stable Dispersal Strategies. IIASA Interim Report IR-99-019 (1999). Proceedings of the Royal Society of London Series B 268:499$508(2001)$
No. 37 Gyllenberg M, Metz JAJ: On Fitness in Structured Metapopulations. IIASA Interim Report IR-99-037 (1999). Journal of Mathematical Biology 43:545-560 (2001).

No. 38 Meszéna G, Metz JAJ: Species Diversity and Population Regulation: The Importance of Environmental Feedback Dimensionality. IIASA Interim Report IR-99-045 (1999).

No. 39 Kisdi É, Geritz SAH: Evolutionary Branching and Sympatric Speciation in Diploid Populations. IIASA Interim Report IR-99-048 (1999).

No. 40 Ylikarjula J, Heino M, Dieckmann U: Ecology and Adaptation of Stunted Growth in Fish. IIASA Interim Report IR-99-050 (1999). Evolutionary Ecology 13:433-453 (1999).

No. 41 Nowak MA, Sigmund K: Games on Grids. IIASA Interim Report IR-99-038 (1999). Dieckmann U, Law R, Metz JAJ (eds): The Geometry of Ecological Interactions: Simplifying Spatial Complexity, Cambridge University Press, Cambridge, UK, pp. 135-150 (2000).

No. 42 Ferrière R, Michod RE: Wave Patterns in Spatial Games and the Evolution of Cooperation. IIASA Interim Report IR-99-041 (1999). Dieckmann U, Law R, Metz JAJ (eds): The Geometry of Ecological Interactions: Simplifying Spatial Complexity, Cambridge University Press, Cambridge, UK, pp. 318-332 (2000).

No. 43 Kisdi É, Jacobs FJA, Geritz SAH: Red Queen Evolution by Cycles of Evolutionary Branching and Extinction. IIASA Interim Report IR-00-030 (2000). Selection 2:161176 (2001).

No. 44 Meszéna G, Kisdi É, Dieckmann U, Geritz SAH, Metz JAJ: Evolutionary Optimisation Models and Matrix Games in the Unified Perspective of Adaptive Dynamics. IIASA Interim Report IR-00-039 (2000). Selection 2:193-210 (2001).

No. 45 Parvinen K, Dieckmann U, Gyllenberg M, Metz JAJ: Evolution of Dispersal in Metapopulations with Local Density Dependence and Demographic Stochasticity. IIASA Interim Report IR-00-035 (2000).

No. 46 Doebeli M, Dieckmann U: Evolutionary Branching and Sympatric Speciation Caused by Different Types of Ecological Interactions. IIASA Interim Report IR-00-040 (2000). The American Naturalist 156:S77-S101 (2000).

No. 47 Heino M, Hanski I: Evolution of Migration Rate in a Spatially Realistic Metapopulation Model. IIASA Interim Report IR-00-044 (2000). The American Naturalist 157:495511 (2001).

No. 48 Gyllenberg M, Parvinen K, Dieckmann U: Evolutionary Suicide and Evolution of Dispersal in Structured Metapopulations. IIASA Interim Report IR-00-056 (2000). Journal of Mathematical Biology 45:79-105 (2002).

No. 49 van Dooren TJM: The Evolutionary Dynamics of Direct Phenotypic Overdominance: Emergence Possible, Loss Probable. IIASA Interim Report IR-00-048 (2000). Evolution 54: 1899-1914 (2000).

No. 50 Nowak MA, Page KM, Sigmund K: Fairness Versus Reason in the Ultimatum Game. IIASA Interim Report IR00-57 (2000). Science 289:1773-1775 (2000).

No. 51 de Feo O, Ferrière R: Bifurcation Analysis of Population Invasion: On-Off Intermittency and Basin Riddling. IIASA Interim Report IR-00-074 (2000). International Journal of Bifurcation and Chaos 10:443-452 (2000). 
No. 52 Heino M, Laaka-Lindberg S: Clonal Dynamics and Evolution of Dormancy in the Leafy Hepatic Lophozia Silvicola. IIASA Interim Report IR-01-018 (2001). Oikos 94:525-532 (2001).

No. 53 Sigmund K, Hauert C, Nowak MA: Reward and Punishment in Minigames. IIASA Interim Report IR-01-031 (2001). Proceedings of the National Academy of Sciences of the USA 98:10757-10762(2001).

No. 54 Hauert C, De Monte S, Sigmund K, Hofbauer J: Oscillations in Optional Public Good Games. IIASA Interim Report IR-01-036 (2001).

No. 55 Ferrière R, Le Galliard J: Invasion Fitness and Adaptive Dynamics in Spatial Population Models. IIASA Interim Report IR-01-043 (2001). Clobert J, Dhondt A, Danchin E, Nichols J (eds): Dispersal, Oxford University Press, pp. 57-79 (2001).

No. 56 de Mazancourt C, Loreau M, Dieckmann U: Can the Evolution of Plant Defense Lead to Plant-Herbivore Mutualism. IIASA Interim Report IR-01-053 (2001). The American Naturalist 158: 109-123 (2001).

No. 57 Claessen D, Dieckmann U: Ontogenetic Niche Shifts and Evolutionary Branching in Size-Structured Populations. IIASA Interim Report IR-01-056 (2001). Evolutionary Ecology Research 4:189-217 (2002).

No. 58 Brandt H: Correlation Analysis of Fitness Landscapes. IIASA Interim Report IR-01-058 (2001).

No. 59 Dieckmann U: Adaptive Dynamics of Pathogen-Host Interacations. IIASA Interim Report IR-02-007 (2002). Dieckmann U, Metz JAJ, Sabelis MW, Sigmund K (eds): Adaptive Dynamics of Infectious Diseases: In Pursuit of Virulence Management, Cambridge University Press, Cambridge, UK, pp. 39-59 (2002).

No. 60 Nowak MA, Sigmund K: Super- and Coinfection: The Two Extremes. IIASA Interim Report IR-02-008 (2002). Dieckmann U, Metz JAJ, Sabelis MW, Sigmund K (eds): Adaptive Dynamics of Infectious Diseases: In Pursuit of Virulence Management, Cambridge University Press, Cambridge, UK, pp. 124-137 (2002).

No. 61 Sabelis MW, Metz JAJ: Perspectives for Virulence Management: Relating Theory to Experiment. IIASA Interim Report IR-02-009 (2002). Dieckmann U, Metz JAJ, Sabelis MW, Sigmund K (eds): Adaptive Dynamics of Infectious Diseases: In Pursuit of Virulence Management, Cambridge University Press, Cambridge, UK, pp. 379-398 (2002).

No. 62 Cheptou P, Dieckmann U: The Evolution of SelfFertilization in Density-Regulated Populations . IIASA Interim Report IR-02-024 (2002). Proceedings of the Royal Society of London Series B 269:1177-1186 (2002).
No. 63 Bürger R: Additive Genetic Variation Under Intraspecific Competition and Stabilizing Selection: A Two-Locus Study. IIASA Interim Report IR-02-013 (2002). Theoretical Population Biology 61:197-213 (2002).

No. 64 Hauert C, De Monte S, Hofbauer J, Sigmund K: Volunteering as Red Queen Mechanism for Co-operation in Public Goods Games. IIASA Interim Report IR-02-041 (2002). Science 296:1129-1132 (2002).

No. 65 Dercole F, Ferrière R, Rinaldi S: Ecological Bistability and Evolutionary Reversals under Asymmetrical Competition. IIASA Interim Report IR-02-053 (2002). Evolution 56:1081-1090 (2002).

No. 66 Dercole F, Rinaldi S: Evolution of Cannibalistic Traits: Scenarios Derived from Adaptive Dynamics. IIASA Interim Report IR-02-054 (2002). Theoretical Population Biology 62:365-374 (2002).

No. 67 Bürger R, Gimelfarb A: Fluctuating Environments and the Role of Mutation in Maintaining Quantitative Genetic Variation. IIASA Interim Report IR-02-058 (2002). Genetical Research 80:31-46 (2002).

No. 68 Bürger R: On a Genetic Model of Intraspecific Competition and Stabilizing Selection. IIASA Interim Report IR02-062 (2002). Amer. Natur. 160:661-682 (2002).

No. 69 Doebeli M, Dieckmann U: Speciation Along Environmental Gradients. IIASA Interim Report IR-02-079 (2002). Nature 421:259-264 (2003).

No. 70 Dercole F, Irisson J, Rinaldi S: Bifurcation Analysis of a Prey-Predator Coevolution Model. IIASA Interim Report IR-02-078 (2002).

No. 71 Le Galliard J, Ferrière R, Dieckmann U: The Adaptive Dynamics of Altruism in Spatially Heterogeneous Populations. IIASA Interim Report IR-03-006 (2003).

No. 72 Taborsky B, Dieckmann U, Heino M: Unexpected Discontinuities in Life-History Evolution Under SizeDependent Mortality. IIASA Interim Report IR-03-004 (2003).

No. 73 Gardmark A, Dieckmann U, Lundberg P: LifeHistory Evolution in Harvested Populations: The Role of Natural Predation. IIASA Interim Report IR-03-008 (2003).

No. 74 Mizera F, Meszena G: Spatial Niche Packing, Character Displacement and Adaptive Speciation Along an Environmental Gradient. IIASA Interim Report IR-03-062 (2003). Evolutionary Ecology Research 5:363-382 (2003).

No. 75 Dercole F: Remarks on Branching-Extinction Evolutionary Cycles. IIASA Interim Report IR-03-077 (2003). Journal of Mathematical Biology 47:569-580 (2003).

Issues of the IIASA Studies in Adaptive Dynamics series can be obtained at www.iiasa.ac.at/Research/ADN/Series.html or by writing to adn@iiasa.ac.at. 


\section{Contents}

1 Introduction 1

2 The model 2

2.1 Ecological model . . . . . . . . . . . . . . . . . . . . . 3

2.2 Evolutionary model . . . . . . . . . . . . . . . . . . . . 4

3 The branching-extinction evolutionary cycle 6

4 Discussion and conclusions $\quad 6$ 


\begin{abstract}
We show in this paper that the evolution of cannibalistic consumer populations can be a never ending story involving alternating levels of polymorphism. More precisely, we show that a monomorphic population can evolve toward high levels of cannibalism until it reaches a so-called branching point, where the population splits into two sub-populations characterized by different, but initially very close, cannibalistic traits. Then, the two traits coevolve until the more cannibalistic subpopulation undergoes evolutionary extinction. Finally, the remaining population evolves back to the branching point, thus closing an evolutionary cycle. The model on which the study is based is purely deterministic and derived through the adaptive dynamics approach. Evolutionary dynamics are investigated through numerical bifurcation analysis, applied both to the ecological (residentmutant) model and to the evolutionary model. The general conclusion emerging from this study is that branching-extinction evolutionary cycles can be present in wide ranges of environmental and demographic parameters, so that their detection is of crucial importance when studying evolutionary dynamics.
\end{abstract}




\author{
About the Author \\ Fabio Dercole \\ CIRITA, Politecnico di Milano \\ Via Ponzio 34/5, 20133 Milano, Italy \\ and \\ Adaptive Dynamics Network \\ International Institute for Applied Systems Analysis \\ A-2361 Laxenburg, Austria
}

\title{
Acknowledgement
}

The author is grateful to Sergio Rinaldi and to three anonymous reviewers for very useful comments and suggestions. The research was supported by MIUR under project FIRB-RBNE01CW3M. 


\title{
Remarks on Branching-Extinction Evolutionary Cycles
}

\author{
Fabio Dercole
}

\section{Introduction}

Red Queen dynamics are evolutionary dynamics that do not converge to an equilibrium (Van Valen, 1973, see also Rosenzweig and Schaffer, 1978, Stenseth and Maynard Smith, 1984, Futuyma, 1986 and Rosenzweig et al., 1987). The name was inspired by the book "Through the LookingGlass and What Alice Found There" (Carroll, 1871), where the Red Queen says: "Now, here, you see, it takes all the running you can do, to keep in the same place." As remarked by the Red Queen, the most intriguing case of Red Queen dynamics is that of evolutionary cycles, where natural selection keep (periodically) the system in the same evolutionary state. This is in contrast with the (wrong) idea that an evolutionary change always implies some sort of improvement.

Evolutionary cycles have captured the attention of theoretical ecologists and geneticists in the last decades (see e.g. Abrams, 1992; Marrow et al., 1992; Dieckmann et al., 1995; Iwasa and Pomiankowski, 1995, 1999; Marrow et al., 1996; Abrams and Matsuda, 1997; Gavrilets, 1997; Dercole et al., 2003). In all the above cited works, the adaptive traits vary cyclically while the population densities track the equilibrium corresponding to the current trait values. Other kinds of evolutionary cycles involve populations which are not at equilibrium at ecological timescale (at least during part of the evolutionary cycle) (Khibnik and Kondrashov, 1997; Dercole et al., 2002b), or switch between different attractors begetting evolutionary reversals (Khibnik and Kondrashov, 1997; Doebeli and Ruxton, 1997; Dercole et al., 2002a). Finally, there is also the possibility of evolutionary cycles due to alternating levels of polymorphism. Such cycles, called branchingextinction evolutionary cycles, are characterized by recurrent evolutionary branching and extinction, which periodically add and remove a population (or morphs) to and from the system.

At a branching point (Metz et al., 1996; Geritz et al., 1997, 1998) one of the resident populations, characterized by a particular trait value, coexists with a population of mutants characterized by a slightly different trait value. Moreover, the two initially similar traits are under opposite selection pressures, so that the mutant population becomes a new resident population and the number of coevolving traits increases. At evolutionary extinction (Matsuda and Abrams, 1994; Ferrière, 2000) the trait of a population reaches a critical value at which the corresponding equilibrium density vanishes or catastrophically collapses to zero, thus reducing the number of coevolving traits. Therefore, in the simplest branching-extinction evolutionary cycle the evolutionary dynamics of a monomorphic population are characterized by a globally stable branching point and the dimorphic evolutionary trajectories originating close to the branching point end with the evolutionary extinction of one of the two sub-populations.

Branching-extinction evolutionary cycles have been observed in several models (Van der Laan and Hogeweg, 1995; Doebeli and Ruxton, 1997; Koella and Doebeli, 1999; Doebeli and Dieckmann, 2000) through stochastic simulations. However, from a stochastic simulation it is hard to say if extinction is produced by demographic stochasticity, when the population density is relatively small, or by the deterministic mechanism of evolutionary extinction described above. Only Kisdi et al. (2001) presented an example of branching-extinction evolutionary cycle where extinction occurs deterministically. Such an example is based on a Lotka-Volterra population competition 
model (Lotka, 1920; Volterra, 1926). As is well known, these models are rather degenerate both biologically (the per capita growth rates are unbounded) and mathematically (their bifurcations are non-generic (Kuznetsov, 1998)). Moreover, the particular model analyzed in Kisdi et al. (2001) uses a very peculiar dependence of the competition coefficients upon the traits. Such a dependence, which is hardly defendable biologically, seems to be adopted simply in view of obtaining a branching-extinction cycle. Finally, mathematically speaking, the long-term evolutionary behavior of the model is not captured by a true cycle. In fact, the dimorphic trajectories originating close to the branching point converge to a point in trait space where both sub-populations go extinct, thus virtually determining the halt of evolutionary dynamics. However, stochastically, one subpopulation goes extinct first. Then, the remaining (very scarce) monomorphic population evolves back to the branching point. Of course, which sub-population goes extinct first is a matter of chance and, depending upon this random event, different monomorphic transients lead back to the branching point. Thus, a stochastic simulation would show long-term evolutionary dynamics in which two different periods (from the branching point back to it) alternate randomly.

In this paper we present the first fully deterministic example of branching-extinction evolutionary cycle, using the model for the evolution of cannibalistic traits in consumer populations recently described in Dercole and Rinaldi (2002). We show that any monomorphic population converges to an intermediate level of cannibalism where it branches into two sub-populations. Then, assuming that body size of adult individuals and cannibalism are positively correlated (as it is often the case (Fox, 1975; Polis, 1981, 1988)), we show that during the dimorphic evolutionary phase the two sub-populations evolve into a weakly cannibalistic $d w a r f$ population and a highly cannibalistic giant population, until the giant population undergoes an evolutionary extinction. The key point of our result is that the giant population density does not vanish gradually at evolutionary timescale, but rather collapses suddenly (Gyllenberg and Parvinen, 2001). Such a discontinuous extinction event reverses the selection pressure on the dwarf population, which then begins to enhance its cannibalistic attitude.

As in Kisdi et al. (2001), we follow the approach of adaptive dynamics theory developed by Metz et al. (1996); Geritz et al. (1997, 1998). This approach is based on the assumption that small and rare random mutations are followed by natural selection and allows one to describe the dynamics of the traits in a purely deterministic way, through an ODE called the canonical equation (Dieckmann and Law, 1996; Champagnat et al., 2001), which is capable of explaining evolutionary branching and extinction.

The paper is organized as follows. In the next section we sketch the derivation of the monomorphic and dimorphic canonical equations. In the third section we derive the branching-extinction evolutionary cycle for a particular parameter setting. A discussion of the mechanisms necessary for this kind of evolutionary cycles to exists and some comments on the robustness of the results close the paper.

\section{The model}

Assume that a cannibalistic consumer population is characterized by a positive phenotypic trait from now on called cannibalism, indicated by $x$ and positively correlated with the size of adult individuals. This assumption is not necessary for our result but it facilitates its interpretation. In fact, $x$ can be simply identified with a suitable measure of adult body size (like length or weight, but see e.g. Mittelbach and Persson (1998) for other examples in cannibalistic fish populations), so that the coexistence of two sub-populations, one with low and one with high cannibalism, should be revealed by the presence of dwarfs and giants in the same environment.

The derivation of the canonical equation of adaptive dynamics requires three things: (i) the knowledge of the interactions occurring at ecological time scale between all sub-populations; (ii) 
the dependence of the demographic parameters of the sub-populations upon the traits; (iii) the frequency and distribution of the mutations. All this is specified in the next two sub-sections.

\subsection{Ecological model}

The interactions between $N$ cannibalistic consumer sub-populations with biomass densities $n_{i}$ and traits $x_{i}, i=1, \ldots, N$, are described by the following ODE:

$$
\dot{n}_{i}=n_{i} f_{i}(n, x)
$$

where

$$
f_{i}(n, x)=\left(\frac{\sum_{j=0}^{N} e_{i j} a_{i j} n_{j}}{1+\sum_{j=0}^{N} h_{i j} a_{i j} n_{j}}-\sum_{j=1}^{N} \frac{a_{j i} n_{j}}{1+\sum_{k=0}^{N} h_{j k} a_{j k} n_{k}}-\sum_{j=1}^{N} c_{i j} n_{j}\right)
$$

$n=\left(n_{1}, \ldots, n_{N}\right), x=\left(x_{1}, \ldots, x_{N}\right), n_{0}$ is the density of a common resource, $a_{i j}$ and $h_{i j}$ are the attack rate and the handling time of the $i$-th sub-population associated with the food source of type $j, e_{i j}$ is a conversion factor transforming food intake of type $j$ into new biomass of type $i$ and $c_{i j}$ specifies the extra-mortality due to competition.

The parameters $n_{0}, e_{i j}$ and $c_{i j}$ are assumed to be constant, while the attack rates $a_{i j}$ and the handling times $h_{i j}$ depend upon the traits as follows:

$$
\begin{gathered}
a_{i 0}=\frac{2 A_{i 0}}{\left(\frac{x_{i}}{x^{0}}\right)^{\alpha}+\left(\frac{x^{0}}{x_{i}}\right)^{\alpha}} \\
a_{i j}=A_{i j}\left[\frac{2}{\left(\frac{p x_{i}}{x_{j}}\right)^{\beta}+\left(\frac{x_{j}}{p x_{i}}\right)^{\beta}}\right]\left(\frac{x_{i}^{\gamma}}{\underline{x}^{\gamma}+x_{i}^{\gamma}}\right)\left(1-\frac{x_{i}^{\delta}}{\bar{x}^{\delta}+x_{i}^{\delta}}\right) \\
h_{i j}=w_{1} x_{i}^{-w_{2}}
\end{gathered}
$$

where $A_{i j}$ is the maximum attack rate, $x^{0}$ is the trait value at which a population is best adapted to the common resource (see eq. (2a)) and $\alpha>1, \beta>1, \gamma>1, \delta>1, p<1, \underline{x}, \bar{x}, w_{1}$ and $w_{2}$ are suitable positive parameters which specify the shape of the functions (2) (see Dercole and Rinaldi, 2002 , for a more detailed description). In particular, the cannibalistic attack rate $a_{i j}$ is higher when the body size of the victim is in a suitable ratio with that of the predator, i.e. when $x_{j}=p x_{i}, p<1$ (see eq. (2b)). Moreover, small values of $\beta$ imply high values of the cannibalistic attack rate $a_{i i}$ (see eq. (2b) with $x_{i}=x_{j}$ ), i.e. great possibilities for individuals of trait $x_{i}$ to predate individuals of the same trait. In the real world such a population would be characterized by a substantial change in size from juvenile to adult, so that adult individuals can easily predate young ones (Polis, 1981, 1988). However, an explicit description of the age/size distribution, which naturally calls for relatively complex age/size structured models (see e.g. Bosch van den et al., 1988; Diekmann et al., 1986; Metz and Diekmann, 1986a,b,c; Briggs et al., 2000; Claessen et al., 2000), poses some problems in the derivation of the canonical equation of adaptive dynamics. In fact, as we shall see in Section 2.2, the canonical equation captures the evolutionary dynamics under the assumptions that an invading mutant generically substitutes its former resident. Unfortunately, as far as we know, this property is not yet proved for structured population models. Moreover, for such models, the canonical equation can hardly be determined in closed form. For these reasons, 
our choice has been to hide the size-structure of the population, thus describing each population with a first order ODE (see eq. (1)) where the parameter $(1 / \beta)$ is a sort of surrogate for the size range of the individuals in the population. As discussed above, $(1 / \beta)$ gives an indication of the level of intra-trait cannibalism, which is enhanced by factors such as, among others, the size range in the population.

\subsection{Evolutionary model}

We now use model $(1,2)$ with $N=2$ and 3 to derive the monomorphic and dimorphic evolutionary models. The first $(N-1)$ populations are considered to be the resident populations while the last population is considered to be the mutant population. Consistently with the adaptive dynamics approach, we assume that mutations are rare events, so that the resident populations are at equilibrium when a mutation occurs and are never challenged by more than one mutation at a time. Moreover, we also assume that the trait $x_{N}$ of the mutant is only slightly different from one of the resident traits, say $x_{i}$ (i.e. $x_{N}=x_{i}+\epsilon$, with $\epsilon$ small) and that the mutant population density $n_{N}$ is initially very small. Under the above conditions, model $(1,2)$ can be used to establish the fate of the mutant and resident populations. Generically, an invading mutant replaces the former resident (see Dercole, 2002, for detailed conditions and proofs) so that, in the end, the system is composed of new set of $(N-1)$ resident populations with $i$-th trait given by $x_{i}+\epsilon$. In the opposite case, i.e. when the mutant population does not invade, it goes extinct so that the traits of the resident populations remain unchanged. This process of mutation and selection can be further specified by making suitable assumptions on the frequency and distribution of small mutations (Dieckmann and Law, 1996; Champagnat et al., 2001). The conclusion for $N=2$ is that the rate at which the trait $x_{1}$ varies at evolutionary time scale is given by the following monomorphic canonical equation:

$$
\dot{x}_{1}=\left.k \bar{n}_{1}\left(x_{1}\right) \frac{\partial \bar{f}_{2}\left(x_{1}, x_{2}\right)}{\partial x_{2}}\right|_{x_{2}=x_{1}}
$$

where $k$ is proportional to the frequency and variance of small mutations, $\bar{n}_{1}\left(x_{1}\right)$ is the resident population equilibrium density in the absence of mutants (see Dercole and Rinaldi, 2002, for a proof of existence and uniqueness) and $\bar{f}_{2}\left(x_{1}, x_{2}\right)$ is the fitness of the mutant, i.e.

$$
\bar{f}_{2}\left(x_{1}, x_{2}\right)=f_{2}\left(\bar{n}_{1}\left(x_{1}\right), 0, x_{1}, x_{2}\right)
$$

Geritz et al. $(1997,1998)$ showed that a stable monomorphic equilibrium $\bar{x}_{1}$ is a branching point if

$$
\left.\frac{\partial^{2} \bar{f}_{2}\left(\bar{x}_{1}, x_{2}\right)}{\partial x_{2}^{2}}\right|_{x_{2}=\bar{x}_{1}}>0
$$

At a branching point a mutant population invades but does not replace the former resident, thus it becomes a new resident itself. Moreover, the two initially very similar traits $x_{1}$ and $x_{2}$ differentiate in accordance with the dimorphic canonical equation (see the forthcoming eq. (6)).

The numerical bifurcation analysis (Kuznetsov, 1998; Doedel et al., 1997; Kuznetsov and Levitin, 1997) of model $(3,4)$ carried out in Dercole and Rinaldi (2002) reveals that a globally stable branching point $\bar{x}_{1}$ characterizes the monomorphic dynamics of a population with wide size range living in a rich environment (i.e. for sufficiently high values of the parameters $1 / \beta$ and $n_{0}$ ). Thus, for such a population, we now focus on the dimorphic evolutionary dynamics, namely the evolution of the cannibalistic traits $x_{1}$ and $x_{2}$ of two coexisting sub-populations with densities $n_{1}$ and $n_{2}$.

As explained in Dercole and Rinaldi (2002), the study of dimorphic evolutionary dynamics must be limited to the coexistence region, which is the region of all pairs $\left(x_{1}, x_{2}\right)$ for which model $(1,2)$, for $N=2$, has a stable and strictly positive equilibrium. Such a region can be 


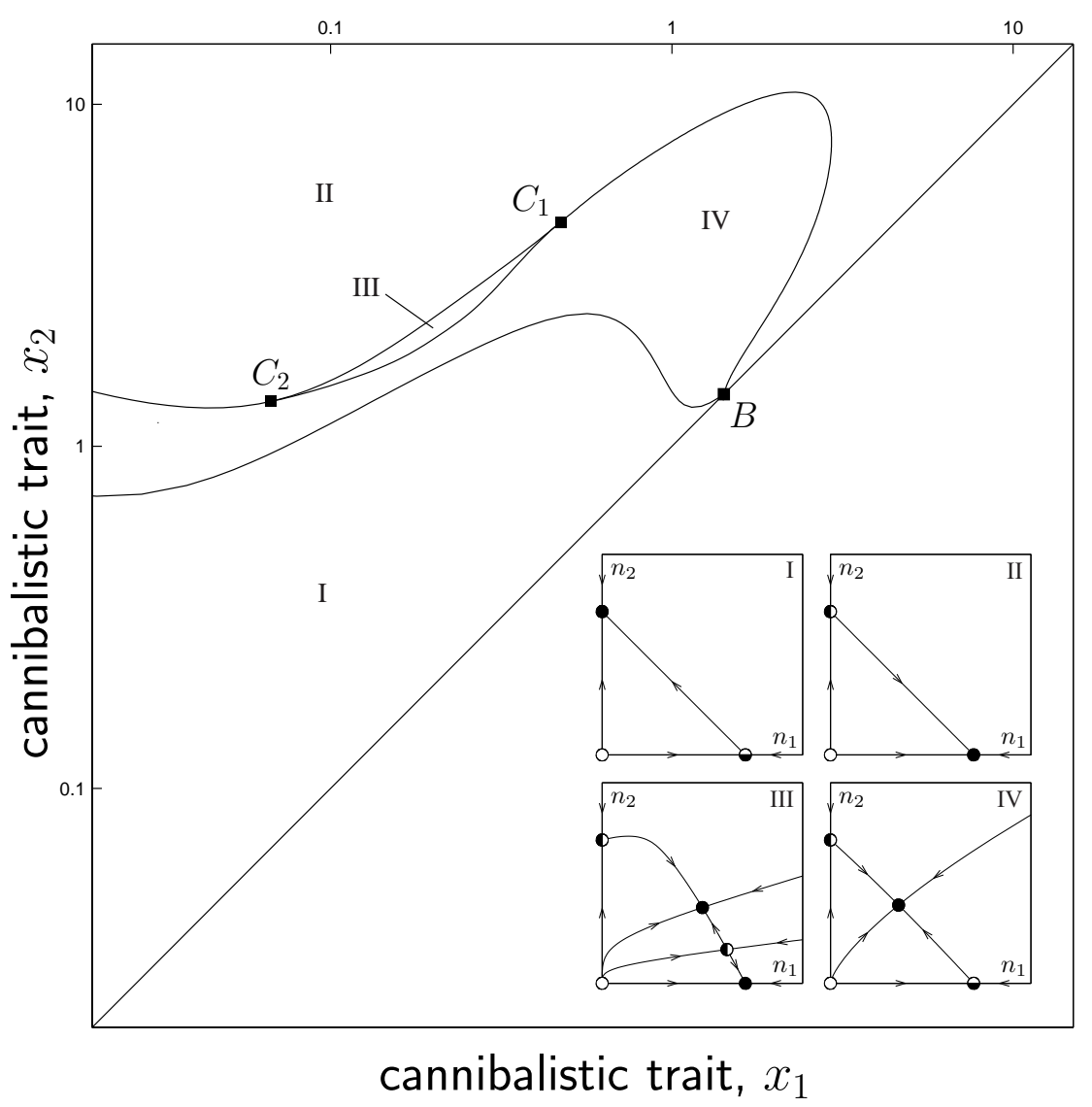

Figure 1: Bifurcation diagram of model $(1,2)(N=2)$ with respect to cannibalistic traits $x_{1}$ and $x_{2}$. Upper triangle: bifurcation curves and regions I-IV (squares indicate codimension-2 bifurcation points). Lower triangle: state portraits of model $(1,2)(N=2)$ for each region I-IV (circles indicate equilibria). Parameter values: $n_{0}=500, \beta=1.9, A_{i 0}=1, A_{i j}=10, x^{0}=0.1$, $\underline{x}=0.5 \bar{x}=5, p=0.2, \alpha=2, \gamma=8, \delta=2, c=1, e=0.6, w_{1}=0.1, w_{2}=0.25, i, j=1,2$. Region III has been horizontally stretched for purpose of illustration.

computed by performing the bifurcation analysis of model $(1,2)(N=2)$ with respect to the traits $x_{1}$ and $x_{2}$ interpreted as constant parameters. Since the trajectories in the space $\left(x_{1}, x_{2}\right)$ are symmetric with respect to the diagonal $x_{2}=x_{1}$, we limit the analysis to the region $x_{1}<x_{2}$ and call populations 1 and 2 dwarf and giant populations, respectively. An example of this bifurcation analysis is shown in Fig. 1, where the upper part reports all bifurcation curves which identify four regions (I-IV), while the lower part reports the corresponding state portraits of model $(1,2)$ $(N=2)$. Since only in the state portraits III and IV there is a stable and strictly positive equilibrium, the region of coexistence is the union of regions III and IV. Point $B$ on the diagonal $x_{2}=x_{1}$ corresponds to the monomorphic branching point, i.e. $B \equiv\left(\bar{x}_{1}, \bar{x}_{1}\right)$. The nature of a bifurcation curve separating two nearby regions can be understood by comparing the two corresponding state portraits. For example, the bifurcation curve separating region II from region IV is characterized (see state portraits II and IV) by the collision of a stable and strictly positive node with a saddle on the $n_{1}$-axis (so-called transcritical bifurcation). Thus, if a dimorphic trajectory in region IV moves toward this bifurcation curve, the giant population density vanishes and the giant population eventually goes extinct when the dimorphic trajectory hits the curve. By contrast, the bifurcation curve separating region II from region III is characterized (see state portraits II and III) 
by the collision of a stable and strictly positive node with a strictly positive saddle (so-called fold bifurcation). Thus, if a dimorphic trajectory in region III moves toward this bifurcation curve, the giant population density does not vanish, but catastrophically collapses (at ecological timescale) as soon as the dimorphic trajectory crosses the curve.

Let now $N=3$ and denote by $\bar{n}_{1}\left(x_{1}, x_{2}\right)$ and $\bar{n}_{2}\left(x_{1}, x_{2}\right)$ the densities of the stable and strictly positive equilibrium of model $(1,2)(N=2)$ in the region of coexistence. The dimorphic canonical equation reads:

$$
\begin{aligned}
& \dot{x}_{1}=\left.k_{1} \bar{n}_{1}\left(x_{1}, x_{2}\right) \frac{\partial \bar{f}_{3}\left(x_{1}, x_{2}, x_{3}\right)}{\partial x_{3}}\right|_{x_{3}=x_{1}} \\
& \dot{x}_{2}=\left.k_{2} \bar{n}_{2}\left(x_{1}, x_{2}\right) \frac{\partial \bar{f}_{3}\left(x_{1}, x_{2}, x_{3}\right)}{\partial x_{3}}\right|_{x_{3}=x_{2}}
\end{aligned}
$$

where $k_{1}$ and $k_{2}$ are proportional to the frequency and variance of small mutations in the two resident populations and $\bar{f}_{3}\left(x_{1}, x_{2}, x_{3}\right)$ is the fitness of the mutant, i.e.

$$
\bar{f}_{3}\left(x_{1}, x_{2}, x_{3}\right)=f_{3}\left(\bar{n}_{1}\left(x_{1}, x_{2}\right), \bar{n}_{2}\left(x_{1}, x_{2}\right), 0, x_{1}, x_{2}, x_{3}\right)
$$

\section{The branching-extinction evolutionary cycle}

The dimorphic evolutionary dynamics defined by model $(6,7)$ within the coexistence region shown in Fig. 1 are sketched in Fig. 2. The coexistence region is partitioned in white, light gray and dark gray sub-regions. Trajectories starting in the white region tend toward a dimorphic equilibrium $D$ (which can be either a branching point or not, see Geritz et al. (1998) or Dercole (2002) for the specific conditions). Trajectories starting in the light gray region hit the boundary of the coexistence region where a catastrophic evolutionary extinction occurs, namely between points $C_{1}$ and $C_{2}$ (see Fig. 1). Notice that points $C_{1}$ and $C_{2}$ are equilibria of equation $(6,7)$. In fact, $\bar{n}_{2}\left(x_{1}, x_{2}\right)=0$ (i.e. $\dot{x}_{2}=0$, see the second equation of model (6)) at such points and $\dot{x}_{1}$ has opposite sign at opposite sides of $C_{1}$ and $C_{2}$ along the boundary of the coexistence region. Thus, a so-called $x_{1}$-nullcline (i.e. a curve in the $\left(x_{1}, x_{2}\right)$ trait space where $\dot{x}_{1}=0$ ) passes through points $C_{1}$ and $C_{2}$. Finally, dark gray regions are those in which the giant [dwarf] population density smoothly vanishes when the evolutionary trajectory approaches the extinction boundary separating region II [I] from region IV (see Fig. 1).

Since the branching point $B$, where dimorphism originates, lies on the boundary of the light gray region the long-term evolutionary attractor of models $(3,4)$ and $(6,7)$ is the branchingextinction evolutionary cycle represented by the thick trajectory in Fig. 2. In words, when dwarf and giant traits become sufficiently different, the giant population is not capable of sustaining itself by harvesting on the dwarf population and is driven extinct by natural selection (see point $X^{*} \equiv$ $\left(x_{1}^{*}, x_{2}^{*}\right)$ in Fig. 2). After that (i.e. after the sudden transition from $X^{*}$ to $X^{* *}$ in Fig. 2) the dwarf population evolves back to the branching point $B$, starting with a trait $x_{1}=x_{1}^{*}$, in accordance with the monomorphic canonical equation $(3,4)$. Thus, starting from any ancestral monomorphic condition the final outcome of evolution is the branching-extinction evolutionary cycle of Fig. 2, characterized by two distinct evolutionary phases: a monomorphic evolution toward the branching point (from $X^{* *}$ to $B$ ) and a dimorphic evolution marked by the temporary presence of a highly cannibalistic population of giants (from $B$ to $X^{*}$ ).

\section{Discussion and conclusions}

An evolutionary cycle characterized by alternating levels of polymorphism has been shown to be the evolutionary attractor of cannibalistic consumer populations with wide size range living in rich 


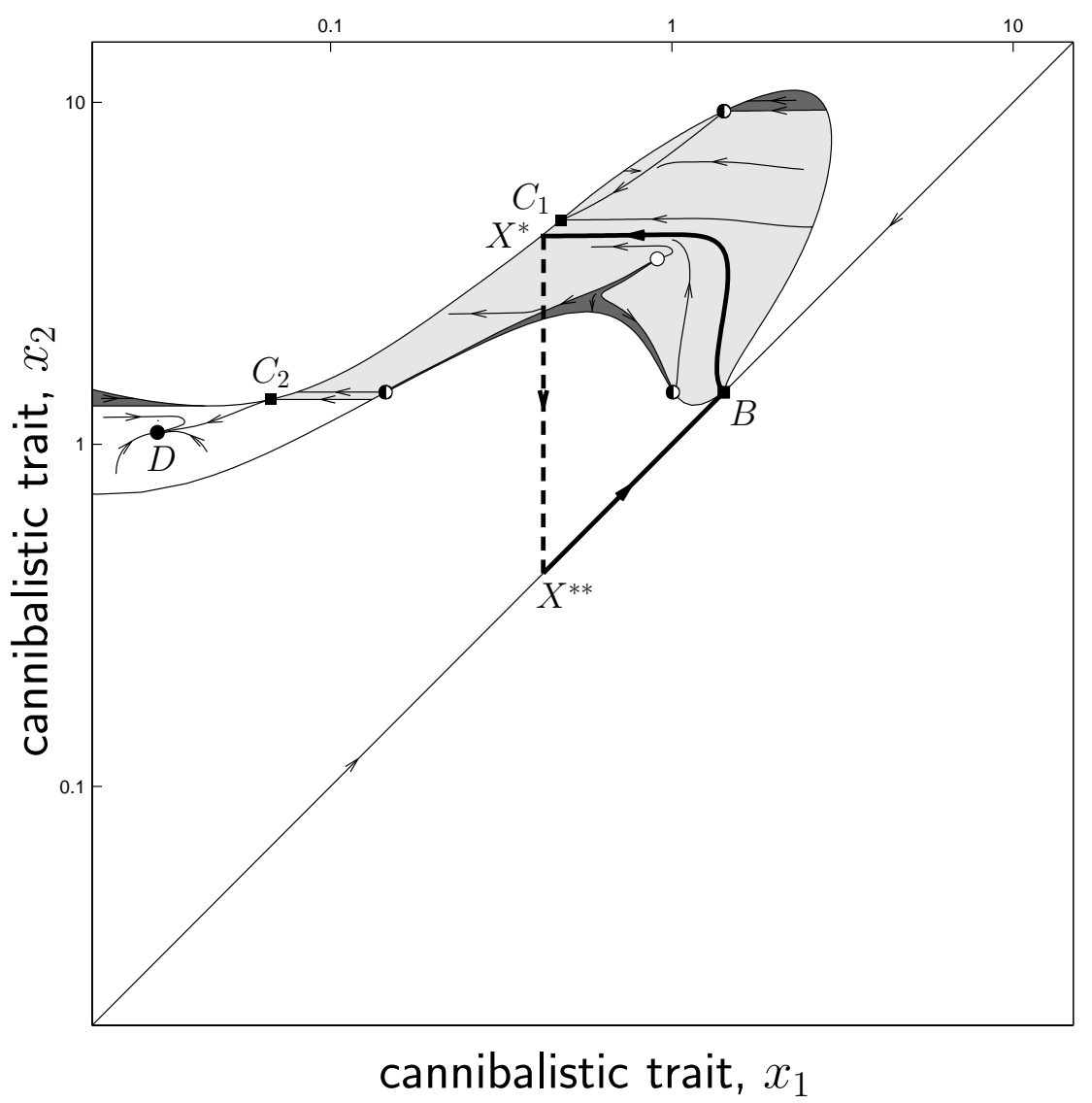

Figure 2: Dimorphic evolutionary dynamics (circles indicate dimorphic equilibria) and the branching-extinction evolutionary cycle (thick trajectory). Parameter values as in Fig. $1\left(k_{1}=k_{2}\right)$.

environments. The deterministic mechanisms that lead to such evolutionary cycles have been first addressed by Kisdi et al. (2001) and require the following three properties: (i) the monomorphic population has an evolutionary branching point where it becomes dimorphic; (ii) the dimorphic evolution originating at the branching point leads to the evolutionary extinction of one of the two morphs, say morph 2; (iii) the post-extinction monomorphic population (i.e. morph 1) is in the basin of attraction of the branching point.

Condition (iii) implies that the direction of evolution of trait 1 reverses during the transition from dimorphism to monomorphism. This is not possible if the evolutionary extinction occurs through a transcritical bifurcation of the dimorphic population equilibrium $\left(\bar{n}_{1}\left(x_{1}, x_{2}\right)\right.$, $\left.\bar{n}_{2}\left(x_{1}, x_{2}\right)\right)$. In fact, in such a case, the population density $\bar{n}_{2}\left(x_{1}, x_{2}\right)$ vanishes when approaching the bifurcation curve, so that only population 1 is present. This implies, by continuity, that $\dot{x}_{1}$ cannot have different values just before and after the bifurcation.

Thus, the key point of our result is that the evolutionary extinction of the giant population occurs through a fold bifurcation, which, being catastrophic, allows the evolutionary reversal of the dwarf population. More precisely, just before the bifurcation, $\dot{x}_{1}$ is negative and given by equation (6) evaluated at point $X^{*}$ (see Fig. 2), where $\bar{n}_{2}\left(x_{1}, x_{2}\right)$ is strictly positive (and equal to the limit of $\bar{n}_{2}\left(x_{1}, x_{2}\right)$ along the evolutionary trajectory approaching $\left.X^{*}\right)$. By contrast, when the evolutionary trajectory crosses the bifurcation curve, the giant population suddenly collapses (i.e. $\bar{n}_{2}\left(x_{1}, x_{2}\right)$ converges to zero at ecological timescale, see state portraits II and III in Fig. 1) and $\dot{x}_{1}$ is positive and given by equation (3) evaluated at point $X^{* *}$. 
Kisdi et al. (2001) have considered a Lotka-Volterra ecological model in which only transcritical bifurcations are possible. Thus, in order to reverse the selection pressure on the remaining population they have been forced to consider a quite peculiar situation in which the evolutionary extinction of both sub-populations occurs simultaneously. This is why in their case the dimorphic evolutionary trajectories converge to a codimension-2 bifurcation point, namely the point of intersection of two transcritical bifurcation curves.

In closing this paper we like to comment on the robustness of the result. In principle, a complete bifurcation analysis of models $(3,4)$ and $(6,7)$ with respect to all couples of strategic parameters (like the environmental richness $\left(n_{0}\right)$ and the size range $(1 / \beta)$ ) would answer all possible questions concerning the robustness of our conclusions. In particular, a complete bifurcation analysis would allow us to determine all possible qualitative evolutionary scenarios and the regions in parameter space where such scenarios occur. However, such an analysis poses nontrivial technical problems, since the dimorphic population equilibrium is not known in closed form, so that model $(6,7)$ is actually a differential algebraic system, for which algorithms for the numerical solution of boundary-value problems are not yet fully developed (Ascher and Spiteri, 1994). Such algorithms are needed for the continuation of heteroclinic bifurcations (saddle to saddle connections) like those present in our model. Indeed, if one would like to determine the boundary of the region in parameter space where the branching-extinction evolutionary cycle exists, one should produce through numerical continuation the parameter combinations for which the unstable manifold of the saddle point $B$ (trajectory $B X^{*}$ ) coincides with the stable manifold of the saddle point $C_{2}$ (see Fig. 2).

Despite these technical difficulties, we checked, by means of extensive numerical integration of model $(6,7)$ for various parameter settings, that the branching-extinction evolutionary cycle of Fig. 2 is structurally stable and present in wide ranges of environmental and demographic parameters. Thus, our conclusion is that branching-extinction evolutionary cycles are robust evolutionary attractors and their detection is of crucial importance for fully understanding evolutionary dynamics.

\section{References}

Abrams, P. A. 1992. Adaptive foraging by predators as a cause of predator-prey cycles. Evol. Ecol. 6, 56-72.

Abrams, P. A. and Matsuda, H. 1997. Prey evolution as a cause of predator-prey cycles. Evolution 51, 1740-1748.

Ascher, U. M. and Spiteri, R. J. 1994. Collocation software for boundary value differentialalgebric equations. SIAM J. Sci. Compt. 15, 938-952.

Bosch van den, F., de Roos, A. M., and Gabriel, W. 1988. Cannibalism as a life boat mechanism. J. Math. Biol. 26, 619-633.

Briggs, C. J., Sait, S. M., Begon, M., Thompson, D. J., and Godfray, H. C. J. 2000. What causes generation cycles in populations of stored product moths? J. Anim. Ecol. 69, 352-365.

Carroll, L. 1871. Through the Looking-Glass and What Alice Found There. MacMillan Press Ltd., London, UK.

Champagnat, N., Ferrière, R., and Ben Arous, G. 2001. The canonical equation of adaptive dynamics: A mathematical view. Selection 2, 73-83. 
Claessen, D., de Roos, A. M., and Persson, L. 2000. Dwarfs and giants: Cannibalism and competition in size-structured populations. Am. Nat. 155, 219-237.

Dercole, F. 2002. Evolutionary Dynamics through Bifurcation Analysis: Methods and Applications. Ph.d. thesis, Department of Electronics and Information, Politecnico di Milano, Milano, Italy.

Dercole, F., Ferrière, R., and Rinaldi, S. 2002a. Ecological bistability and evolutionary reversals under asymmetrical competition. Evolution 56, 1081-1090.

Dercole, F., Gragnani, A., Ferrière, R., and Rinaldi, S. 2002b. Coevolution of slow-fast populations: An application to prey-predator systems (submitted to The American Naturalist).

Dercole, F., Irisson, J-O., and Rinaldi, S. 2003. Bifurcation analysis of a prey-predator coevolution model. SIAM J. App. Math. 63, 1378-1391.

Dercole, F. and Rinaldi, S. 2002. Evolution of cannibalism: Scenarios derived from adaptive dynamics. Theor. Popul. Biol. 62, 365-374.

Dieckmann, U. and Law, R. 1996. The dynamical theory of coevolution: A derivation from stochastic ecological processes. J. Math. Biol. 34, 579-612.

Dieckmann, U., Marrow, U., and Law, R. 1995. Evolutionary cycling in predator-prey interactions: Population dynamics and the Red Queen. J. Theor. Biol. 176, 91-102.

Diekmann, O., Nisbet, R. M., Gurney, W. S. C., and van den Bosch, F. 1986. Simple mathematical models for cannibalism: A critique and a new approach. Math. Biosci. 78, 21-46.

Doebeli, M. and Dieckmann, U. 2000. Evolutionary branching and sympatric speciation caused by different types of ecological interactions. Am. Nat. 156, 77-101.

Doebeli, M. and Ruxton, G. D. 1997. Evolution of dispersal rates in metapopulation models: Branching and cyclic dynamics in phenotype space. Evolution 51, 1730-1741.

Doedel, E., Champneys, A., Fairgrieve, T., Kuznetsov, Y. A., Sandstede, B., and Wang, X. 1997. AUTO97: Continuation and bifurcation software for ordinary differential equations (with HOMCONT). Department of Computer Science, Concordia University, Montreal, QC.

Ferrière, R. 2000. Adaptive responses to environmental threats: Evolutionary suicide, insurance and rescue. Options, journal of the International Institute for Applied Systems Analysis, Laxenburg, Austria Spring 2000, 12-16.

Fox, L. R. 1975. Cannibalism in natural populations. Annu. Rev. Ecol. Syst. 6, 87-106.

Futuyma, D. J. 1986. Evolutionary Biology. Sinauer Associate, Sunderland, MA.

Gavrilets, S. 1997. Coevolutionary chase in exploiter-victim systems with polygemic characters. J. Theor. Biol. 186, 527-534.

Geritz, S. A. H., Kisdi, E., Meszéna, G., and Metz, J. A. J. 1998. Evolutionarily singular strategies and the adaptive growth and branching of the evolutionary tree. Ecology 12, 35-57.

Geritz, S. A. H., Metz, J. A. J., Kisdi, E., and Meszéna, G. 1997. The dynamics of adaptation and evolutionary branching. Phys. Rev. Lett. 78, 2024-2027.

Gyllenberg, M. and Parvinen, K. 2001. Necessary and sufficient conditions for evolutionary suicide. Bull. Math. Biol. 63, 981-993. 
Iwasa, Y. and Pomiankowski, A. 1995. Continual change in mate preferences. Nature 377, 420422.

Iwasa, Y. and Pomiankowski, A. 1999. Good parent and good genes models of handicap evolution. J. Theor. Biol. 200, 97-109.

Khibnik, A. I. and Kondrashov, A. S. 1997. Three mechanisms of Red Queen dynamics. Proc. $R$. Soc. Lond. B 264, 1049-1056.

Kisdi, E., Jacobs, F. J. A., and Geritz, S. A. H. 2001. Red Queen evolution by cycles of evolutionary branching and extinction. Selection 2, 161-176.

Koella, J. C. and Doebeli, M. 1999. Population dynamics and the evolution of virulence in epidemiological models with discrete host generations. J. Theor. Biol. 198, 461-475.

Kuznetsov, Y. A. 1998. Elements of Applied Bifurcation Theory. Springer Verlag, Berlin.

Kuznetsov, Y. A. and Levitin, V. V. 1997. CONTENT: A multiplatform environment for analyzing dynamical systems. Dynamical Systems Laboratory, Centrum voor Wiskunde en Informatica, Amsterdam, The Netherlands available from ftp. cwi.nl/pub/CONTENT.

Lotka, A. J. 1920. Undamped oscillations derived from the law of mass action. J. Am. Chem. Soc. 42, 1595-1599.

Marrow, P., Dieckmann, U., and Law, R. 1996. Evolutionary dynamics of predator-prey systems: An ecological perspective. J. Math. Biol. 34, 556-578.

Marrow, P., Law, R., and Cannings, C. 1992. The coevolution of predator-prey interactions: ESSs and Red Queen dynamics. Proc. R. Soc. Lond. B 250, 133-141.

Matsuda, H. and Abrams, P. A. 1994. Runaway evolution to self-extinction under asymmetrical competition. Evolution 48, 1764-1772.

Metz, J. A. J. and Diekmann, O. 1986a. Age dependence. In The Dynamics of Physiologically Structured Populations (J. A. J. Metz and O. Diekmann, eds.). Springer Verlag, Lecture Notes in Biomathematics, 68, pp. 136-184.

Metz, J. A. J. and Diekmann, O. 1986b. Formulating models for structured populations. In The Dynamics of Physiologically Structured Populations (J. A. J. Metz and O. Diekmann, eds.). Springer Verlag, Lecture Notes in Biomathematics, 68, pp. 78-135.

Metz, J. A. J. and Diekmann, O. 1986c. A gentle introduction to structured population models: Three worked examples. In The Dynamics of Physiologically Structured Populations (J. A. J. Metz and O. Diekmann, eds.). Springer Verlag, Lecture Notes in Biomathematics, 68, pp. 345 .

Metz, J. A. J., Geritz, S. A. H., Meszéna, G., Jacobs, F. J. A., and van Heerwaarden, J. S. 1996. Adaptive dynamics: A geometrical study of the consequences of nearly faithful reproduction. In Stochastic and Spatial Structures of Dynamical Systems (S. J. van Strien and S. M. Verduyn Lunel, eds.). NHA, pp. 183-231.

Mittelbach, G. G. and Persson, L. 1998. The ontogeny of piscivory and its ecological consequences. Can. J. Fish. Aquat. Sci. 55, 1454-1465.

Polis, G. A. 1981. The evolution and dynamics of intraspecific predation. Annu. Rev. Ecol. Syst. 12, 225-251. 
Polis, G. A. 1988. Exploitation competition and the evolution of interference, cannibalism and intraguild predation in age/size-structured populations. In Size-Structured Populations: Ecology and Evolution (B. Ebenman and L. Persson, eds.). Springer, Heidelberg, pp. 185-202.

Rosenzweig, M. L., Brown, J. S., and Vincent, T. L. 1987. Red Queen and ESS: The coevolution of evolutionary rates. Evol. Ecol. 1, 59-94.

Rosenzweig, M. L. and Schaffer, W. M. 1978. Homage to Red Queen II. Coevolutionary response to enrichment of exploitation ecosystems. Theor. Popul. Biol. 14, 158-163.

Stenseth, N. C. and Maynard Smith, J. 1984. Coevolution in ecosystems: Red Queen evolution or stasis. Evolution 38, 870-880.

Van der Laan, J. D. and Hogeweg, P. 1995. Predator-prey coevolution: Interactions across different timescales. Proc. R. Soc. Lond. B 259, 35-42.

Van Valen, L. 1973. A new evolutionary law. Evol. Theory 1, 1-30.

Volterra, V. 1926. Variazioni e fluttuazioni del numero d'individui in specie animali conviventi. Mem. Accad. Naz. Lincei 2, 31-113. 